\title{
Respiratory medicine: what is the diagnosis?
}

\section{To the Editors:}

Clues of the pressurised metered-dose inhaler in the left shirt pocket and the right upper lobe fibrosis should prompt the reader to consider the unifying diagnosis of allergic bronchopulmonary aspergillosis (fig. 1). This 48-yr-old male had difficult-to-control asthma with elevated serum immunoglobulin E levels, presence of aspergillus immunoglobulin E antibodies, and the occurrence of central bronchiectasis, fibrosis and loss of volume in the right upper lobe on computed tomography of the thorax. The common causes of upper lobe fibrosis in the lungs are Tuberculosis, Extrinsic allergic alveolitis, Allergic bronchopulmonary aspergillosis, Radiation, and Sarcoidosis (TEARS). Other causes include ankylosing spondylitis, drugs, silicosis, and Langerhans' cell histiocytosis. The patient responded well to oral corticosteroid therapy and continues to be on maintenance prednisolone 2 yrs following the diagnosis.

D.K.C. Lee

Dept of Respiratory Medicine, Ipswich Hospital, Ipswich, UK.

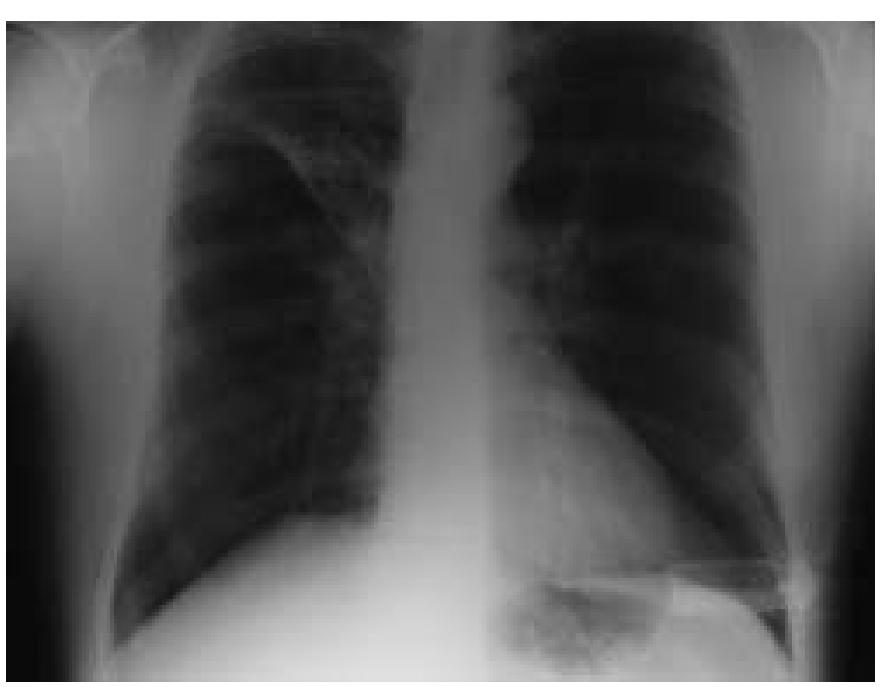

FIGURE 1. Chest radiograph.

\section{Perception of what?}

\section{To the Editors:}

Finding risk factors for severe attacks of asthma is an important issue. The study published in the European Respiratory Journal by BARREIRO et al. [1] is of interest as it concluded that blunted perception of dyspnoea may be of importance. It is summarised that "Dyspnoea at peak exercise is the best indicator of the near-fatal asthma condition". Two small groups of asthmatic patients with $(n=7)$ and without $(n=8)$ a recent nearfatal asthma (NFA) attack ("at least one NFA attack within the 5 yrs previous to study entry" [1]) were compared.

However, the article gives rise to some questions and comments. What was the definition of NFA? No description was given other than that cited above. Did the patients visit an emergency room? How was lung function? How can one know that the attacks were near fatal without examining consciousness, cyanosis, carbon dioxide retention, or by other appropriate tests?

It is well documented in several studies that symptoms generally attributed to asthma are not well correlated (sometimes not at all) to lung function values, such as forced expiratory volume in one second (FEV1). For this reason, how can one expect that dyspnoea or perception of dyspnoea (which is one symptom) could be relevant for bronchial obstruction and severe asthma attack? What is the hypothesis?
Some of the (believed) asthma symptoms are not indicators of bronchial obstruction (asthma) but of asthma-like disorders [2$5]$, such as sensory hyperreactivity $[4,6]$. These disorders are often mixed up with classical asthma [5] and a patient may also have classical asthma and sensory hyperreactivity simultaneously $[4,6]$. There are reasons to suspect that perception of dyspnoea has a very limited direct association with bronchial obstruction (asthma); more important may be a disturbance of the breathing regulation. Low lung function values in these patients may sometimes be interpreted as asthma, but may in fact be better explained by an inability to perform a forced expiration, e.g. when recording FEV1 and peak expiratory flow.

Exercise may induce bronchial obstruction (exercise-induced bronchial obstruction), but may also induce symptoms that are similar to those in asthma/bronchial obstruction [7]. This is often overlooked in the literature. If one likes to predict the risk for acute bronchial asthma, such as NFA, and use a physical test, it may be logical to also document the degree of bronchial obstruction after exercise, e.g. changes in FEV1. Where are those data? What was the correlation between the degree of dyspnoea and lung function before, during and after exercise?

Thus, there are several basic questions that have to be answered before it may be concluded that perception of dyspnoea is of any importance for the prediction of severe asthma attacks. 


\section{O. Löwhagen}

Asthma and Allergy Centre, Sahlgrenska University Hospital, Göteborg, Sweden.

\section{REFERENCES}

1 Barreiro E, Gea J, Sanjuás C, Marcos R, Broquetas J, MilicEmili J. Dyspnoea at rest and at the end of different exercises in patients with near-fatal asthma. Eur Respir J 2004; 24: 219-225.

2 Lowhagen O. Functional respiratory disorder as a significant differential diagnosis in asthma. Lakartidningen 1989; 86: 57.

3 Ringsberg KC, Lowhagen O, Sivik T. Psychological differences between asthmatics and patients suffering from an asthma-like condition, functional breathing disorder. Integr Physiol Behav Sci 1993; 28: 358-367.

4 Millqvist E, Bende M, Lowhagen O. Sensory hyperreactivity - a possible mechanism underlying cough and asthmalike symptoms. Allergy 1998; 53: 1208-1212.

5 Lowhagen O. Asthma and asthma-like disorders. Review Respir Med 1999; 93: 851-855.

6 Ternesten-Hasseus E, Farbrot A, Löwhagen O, Millqvist E. Sensitivity to methacholine and capsaicin in patients with unclear respiratory symptoms. Allergy 2002; 57: 501-507.

7 Löwhagen O, Arvidsson M, Bjarneman P, Jorgensen N. Exercise-induced respiratory symptoms are not always asthma. Respir Med 1999; 93: 734-738.

DOI: $10.1183 / 09031936.05 .00124804$

From the authors:

We would like to thank O. Löwhagen for his comments and suggestions on our recent article published in the European Respiratory Journal [1]. We shall try to respond to and clarify his doubts to the best of our ability.

Regarding the definition of "near-fatal asthma" (NFA), we have to recognise that, due to the space limitations, this was not extensively covered in the final version of the paper. However, we did devote at least eight lines to defining this group (page 219, last two lines, and page 210, first six lines). NFA criteria were those established by a panel of experts from 10 different countries participating in a multinational project, the European Network for Understanding the Mechanisms of Severe Asthma (ENFUMOSA; ref BMH4-96-1417, EC) [2], the purpose of which, accordingly, was to investigate, in depth, the clinical, physiological and biological mechanisms of severe asthma. O. Löwhagen can find more details in a previous paper of the ENFUMOSA study group [3]. With the same concern in mind, our colleague asks about other details, such as whether the patients have visited an emergency room or what their lung function was like. If he re-reads the paper carefully he will notice that "all patients had been hospitalised due to asthma" (page 219, lines 2 and 3) and the NFA "had a history of at least one NFA attack" (which of course implies at least one visit to an emergency room) (page 219, lines 4-6). Lung function is specifically referred to in table 1. Regarding a wider definition of NFA attack, we transcribe here the agreement of the ENFUMOSA panel: "a respiratory arrest and/or $\mathrm{Pa}, \mathrm{CO}_{2}<50 \mathrm{mmHg}^{\prime \prime}$ [4].
O. Löwagen also asks for the hypothesis, but this is specifically provided in the paper (page 219, Introduction section, lines 1923): "...those patients who have experienced NFA should report a lower perception of dyspnoea". We agree with our colleague that "some of the believed asthma symptoms are not indicators of bronchial obstruction"; however. we disagree with both the synonymy he establishes between "airway obstruction" and "asthma", and the splicing of the current concept of asthma into many other subconcepts. The accepted definition of asthma is "...a chronic inflammatory disorder of the airways in which many cells and cellular elements play a role. The chronic inflammation causes an associated increase in airway responsiveness that leads to recurrent episodes of wheezing, breathlessness, chest tightness, and coughing, particularly at night or in the early morning. These episodes are usually associated with widespread but variable airflow obstruction that is often reversible either spontaneously or with treatment" [5]. The presence of a disturbance of the breathing regulation does not exclude, in any case, the diagnosis of asthma in the same way that airway obstruction is not equivalent to asthma.

Regarding functional data following exercise, these are not included in the paper since it was not one of the objectives. However, determinations of static lung volumes, specific airway resistance (SRaw) and maximal inspiratory pressures were performed at the end of all exercises, since a complementary study was conducted to investigate the effects of different efforts on muscle and lung function. Significant changes in SRaw were observed following general exercise and the threshold inspiratory loading test [6]. Unfortunately, we were not able to also record lung function "during the exercise", as suggested by the reviewer.

We trust these answers will have served to convince our colleague.

\section{J. Gea, E. Barreiro and C. Sanjuas}

Servei Pneumologia - URMAR, Hospital del Mar - IMIM, CEXS Dept, UPF, Barcelona, Spain.

\section{REFERENCES}

1 Barreiro E, Gea J, Sanjuás C, Marcos R, Broquetas J, MilicEmili J. Dyspnoea at rest and at the end of different exercises in patients with near-fatal asthma. Eur Respir J 2004; 24: 219-225.

2 http: / / ica.cordis.lu / search / index.cfm? fuseaction $=$ proj. simpledocument \&PJ_RCN $=2683586 \& C F I D=1201809 \&$ CFTOKEN $=40685309$.

3 The ENFUMOSA study group. The ENFUMOSA crosssectional European multicentre study of the clinical phenotype of chronic severe asthma. Eur Respir J 2003; 22: 470-477.

4 ENFUMOSA Study Group. Proceeding book. Section 1: Patient Protocol. London, ENFUMOSA, 1997; pp. 13.

5 Global initiative for Asthma (GINA), 2003 update. www.ginasthma.com.

6 Barreiro E, Gea J, Sanjuás C, et al. Lung volumes and respiratory muscle function before and after exercise in patients with severe asthma (SA). Eur Respir J 1998; 12: Suppl. 29, 64s-65s.

DOI: $10.1183 / 09031936.05 .00137404$ 